\title{
Successful Dupilumab Treatment of Atopic Dermatitis in a Liver Transplant Patient
}

\author{
Amanda Krenitsky, $\mathrm{MD}^{1}$, Sairekha Ravichandran, $\mathrm{MD}^{1,2}$, Joel Cohen, $\mathrm{BS}^{1}$ Jonathan Braue, $\mathrm{MD}^{3}$, \\ Lilia Correa-Selm, MD ${ }^{1,2}$ \\ ${ }^{1}$ University of South Florida, Department of Dermatology and Cutaneous Surgery, Tampa, FL \\ ${ }^{2} \mathrm{H}$. Lee Moffitt Cancer Center, Department of Cutaneous Oncology, Tampa, FL \\ ${ }^{3}$ Scully Welsh Cancer Center, Cleveland Clinic Indian River Hospital, Department of Dermatology and Cutaneous \\ Oncology, Vero Beach, FL
}

\section{ABSTRACT}

Atopic dermatitis (AD) in liver transplant (LT) recipients is well-documented, despite frequent use of immunosuppressants in these patients. In a liver transplant population, the prevalence of $A D$ in children and adults is $27.6 \%$ and $10.3 \%$, respectively. ${ }^{1}$ However, there is dearth of literature regarding treatment of this population, and standard regimens are not well-defined. ${ }^{2-4}$ Here, we report a case of successful treatment of $A D$ in an orthotopic LT patient with dupilumab.

\section{INTRODUCTION}

Atopic dermatitis (AD) in liver transplant (LT) recipients is well-documented, despite frequent use of immunosuppressants in these patients. In a liver transplant population, the prevalence of $A D$ in children and adults is $27.6 \%$ and $10.3 \%$, respectively. ${ }^{1}$ However, there is little literature regarding treatment of this population, and standard regimens are not well-defined. ${ }^{2-4}$ Here, we report a case of successful treatment of $A D$ in an orthotopic LT patient with dupilumab.

\section{CASE REPORT}

A 65-year-old Caucasian female presented with 2-year history of pruritic eruption involving the extremities. Medical history was significant for hypertension, gastroesophageal reflux, and orthotopic liver transplantation 17 years prior for which she was receiving tacrolimus. There was no known personal, family, or donor history of atopy. Physical examination revealed pruritic, hyperkeratotic, scaly papules and lichenified plaques involving flexural surfaces of the bilateral upper and lower extremities. Biopsy revealed spongiotic dermatitis with eosinophils, consistent with atopic dermatitis versus allergic contact dermatitis versus other ID reaction. Patch testing was positive for methyldibromo glutaronitrile, benzalkonium chloride, and benzophenone.

Despite strict allergen avoidance and trials of varying-potency topical, intramuscular, and oral corticosteroids, the patient experienced continued pruritus and repeated flares. Phototherapy was relatively contraindicated secondary to patch-test proven concomitant photoallergic contact dermatitis. Furthermore, use of immunosuppressants such as methotrexate March 2022 Volume 6 Issue 2 
and cyclosporine were contraindicated in this patient, due to LT status with concern for additional hepatotoxic agents and her pre-existing hypertension, respectively.

After discussion with the patient's care team, the patient was started on a dupilumab regimen for persistent atopic dermatitis, with an initial loading dose of 600 milligrams followed by 300 milligrams every two weeks. Complete clearance of the rash, reduced flares, and ninety percent improvement in subjective pruritus were noted within one month and sustained throughout two additional years of treatment. The hepatic function panel and immunosuppressant levels were monitored monthly for three months, then every three months after initiation of dupilumab. Treatment was welltolerated and no issues with hepatic function, immunosuppressant levels, or graft-related complications were reported.

\section{DISCUSSION}

Liver transplant portends a significantly higher risk of post-transplant allergy, autoimmunity, and immune mediated disorders than other solid-organ transplants (40\% vs $1.3 \%){ }^{7}$ Exaggerated Th2-mediated inflammation secondary to suppression of the Th1 immune pathway by calcineurin inhibitors (i.e., tacrolimus) is one proposed mechanism. To this regard, inhibition of the Th2 response may offer a promising approach to the treatment of these patients.

Dupilumab, a monoclonal antibody that decreases Th2-mediated inflammation by selectively blocking IL-4 and IL-13 signaling, has become an important component in the management of inflammatory and autoimmune diseases. Additionally, dupilumab enhances skin repair by increasing epidermal barrier proteins. ${ }^{5}$ ince FDA approval, the use of dupilumab in the treatment of $A D$ is well-established, with fewer contraindications and a more favorable side effect profile than traditional immunosuppressants. 5,6 While there are cases reporting safe and efficacious treatment of AD with dupilumab in liver, heart, and kidney transplant patients, its use in this setting has not been studied in clinical trials. ${ }^{2-4}$

\section{CONCLUSION}

We present a case of dupilumab treatment of $A D$ with superimposed allergic contact dermatitis in an orthotopic LT patient in the setting of posttransplant immunosuppressive therapy, demonstrating favorable clinical response and good side effect profile, maintained for two subsequent years. Prior to the dupilumab trial, this patient's condition was refractory to several treatments despite considerable immunosuppression with transplant medications. As such, dupilumab may be considered a viable treatment option among solid-organ transplant patients with $A D$ unresponsive to topical treatments, phototherapy, and traditional immunosuppressants.

Conflict of Interest Disclosures: Dr. Correa is a consultant for Accutec Blades

Funding: None

Corresponding Author:

Amanda Krenitsky, MD

University of South Florida,

12901 Bruce B Downs BLVD

Tampa, FL 33612

Phone: 813-493-3034

Fax : 813-974-4272

Email: akrenitsky1@usf.edu

\section{References:}

1. Topal E, Çatal F, Selimoğlu MA, et al. Acquired atopic disease after liver transplantation in children; similarities to and differences from adults: a preliminary study. Eur J Gastroenterol March 2022 Volume 6 Issue 2 
Hepatol. Sep 2014;26(9):1055-9.

doi:10.1097/meg.0000000000000142

2. Ludriksone L, Elsner P, Malessa C, Settmacher

$U$, Schliemann S. Effectiveness and safety of dupilumab for atopic dermatitis in a liver transplant recipient: a case report. $J$ Dtsch Dermatol Ges. Jul 2020;18(7):740-742. doi:10.1111/ddg.14074

3. Kha C, Raji K, Chisolm S. Treatment of Atopic Dermatitis With Dupilumab in a Renal Transplant Patient. Dermatitis. Mar/Apr 2020;31(2):e17-e18. doi:10.1097/der.0000000000000560

4. Jamgochian M, Milgraum D, Milgraum S, Pappert A. Dupilumab as treatment for atopic dermatitis in a pediatric heart transplant patient: A case report. Dermatol Ther. Nov 2020;33(6):e14018. doi:10.1111/dth.14018

5. Tsianakas A, Ständer S. Dupilumab: a milestone in the treatment of atopic dermatitis. Lancet. Jan 2 2016;387(10013):4-5. doi:10.1016/s01406736(15)00389-x

6. Product Approval Information - Licensing Action Dupixent (dupilumab). Available online at: https://www.accessdata.fda.gov/drugsatfda_docs /label/2017/761055lbl.pdf (accessed on 06 Sep 2021).

7. Marcus N, Amir AZ, Grunebaum E, et al. De Novo Allergy and Immune-Mediated Disorders Following Solid-Organ Transplantation-

Prevalence, Natural History, and Risk Factors. $J$ Pediatr. May 2018;196:154-160.e2.

doi:10.1016/j.jpeds.2017.11.026 